\title{
Evaluation of parasite subpopulations and genetic diversity of the msp1, msp2 and glurp genes during and following artesunate monotherapy treatment of Plasmodium falciparum malaria in Western Cambodia
}

Panita Gosi ${ }^{1}$, Charlotte A Lanteri ${ }^{1}$, Stuart D Tyner ${ }^{1}$, Youry Se${ }^{2}$, Chanthap Lon ${ }^{2}$, Michele Spring ${ }^{1}$, Mengchuor Char ${ }^{3}$, Darapiseth Sea², Sabaithip Sriwichai ${ }^{2}$, Sittidech Surasri ${ }^{1}$, Saowaluk Wongarunkochakorn ${ }^{1}$, Kingkan Pidtana', Douglas S Walsh', Mark M Fukuda', Jessica Manning ${ }^{1}$, David L Saunders ${ }^{1}$ and Delia Bethell ${ }^{1 *}$

\begin{abstract}
Background: Despite widespread coverage of the emergence of artemisinin resistance, relatively little is known about the parasite populations responsible. The use of PCR genotyping around the highly polymorphic Plasmodium falciparum msp1, msp2 and glurp genes has become well established both to describe variability in alleles within a population of parasites, as well as classify treatment outcome in cases of recurrent disease. The primary objective was to assess the emergence of minority parasite clones during seven days of artesunate (AS) treatment in a location with established artemisinin resistance. An additional objective was to investigate whether the classification of clinical outcomes remained valid when additional genotyping was performed.

Methods: Blood for parasite genotyping was collected from 143 adult patients presenting with uncomplicated falciparum malaria during a clinical trial of AS monotherapy in Western Cambodia. Nested allelic type-specific amplification of the genes encoding the merozoite surface proteins 1 and 2 (msp1 and msp2) and the glutamate-rich protein (g/urp) was performed at baseline, daily during seven days of treatment, and again at failure. Allelic variants were analysed with respect to the size of polymorphisms using Quantity One software to enable identification of polyclonal infections.

Results: Considerable variation of msp2 alleles but well-conserved msp1 and glurp were identified. At baseline, 31\% of infections were polyclonal for one or more genes. Patients with recurrent malaria were significantly more likely to have polyclonal infections than patients without recurrence (seven of nine versus 36 of $127, p=0.004$ ). Emergence of minority alleles during treatment was detected in only one of twenty-three cases defined as being artemisinin resistant. Moreover, daily genotyping did not alter the final outcome classification in any recurrent cases. (Continued on next page)
\end{abstract}

\footnotetext{
* Correspondence: delia.bethell@afrims.org

'Department of Immunology and Medicine, Armed Forces Research Institute of Medical Science (AFRIMS), Bangkok, Thailand

Full list of author information is available at the end of the article
} 


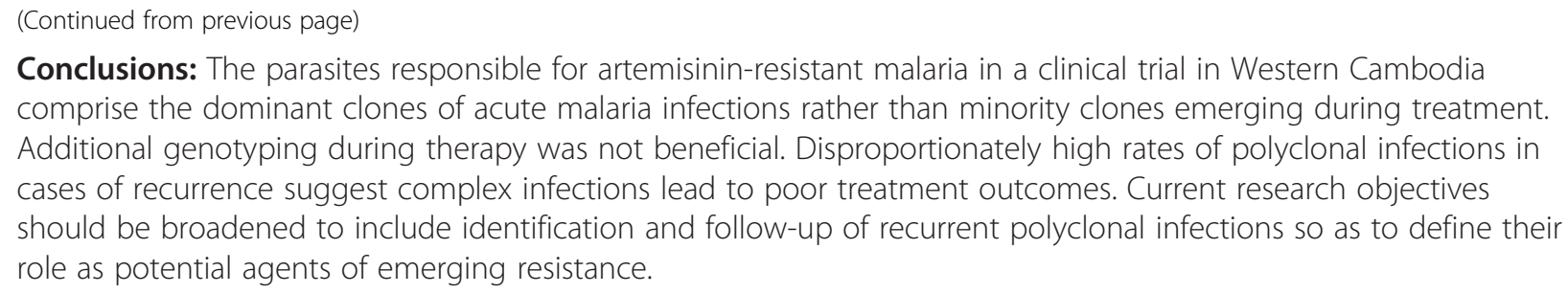

Keywords: Malaria, Plasmodium falciparum, Cambodia, Genotype, Artesunate, msp1, msp2, glurp

\section{Background}

With the emergence of artemisinin resistance in Western Cambodia and along its international border with Thailand as well as further afield in Vietnam and along Thailand's western border with Myanmar, there is a public health mandate to characterize the parasite populations responsible for poor clinical outcomes, as well as better understand the dynamics of how these parasite clones co-exist [1-3]. Gene flow studies and complex whole genome sequencing analyses offer highly sophisticated approaches and have already been employed in this region in an attempt to define the aetiology of the emergence and spread of resistance $[4,5]$. However these approaches are costly and require considerable expertise both to analyse samples and interpret the data.

The use of polymerase chain reaction (PCR) genotyping around a limited number of polymorphic genes, which are known to be highly variable in Plasmodium falciparum parasites, has become well established both to describe variability in alleles within a population of parasites, as well as to distinguish recrudescence from new infection in cases of recurrent disease [6-8]. This technique is relatively cost-efficient and straightforward to perform without requiring extensive sequencing and bioinformatics capabilities. The technique provides a basic description of parasite populations and diversity within a patient cohort using these highly variable parasite genes, as well as classification of recurrent malaria episodes as recrudescent (true failures) or new infections. The most widely used PCR genotyping assay involves nested allelic type-specific amplification of the genes encoding the merozoite surface proteins 1 and 2 ( $m s p 1$ and $m s p 2$ ) and the glutamate-rich protein (glurp) $[8,9]$. This method is highly sensitive and allows for classification of allelic variants including minor variants in mixed clonal infections [10]. The use of high-resolution methods, such as repeated agarose gel electrophoresis with computer image software, for determining DNA band size has increased the ability to distinguish and analyse PCR products that differ only slightly in size and thus have improved the accuracy of this technique. MSP1 block 2 allelic variants fall under three major types - MAD20, K1 and RO33. The msp2 gene can be similarly grouped into two allelic families, FC27 and IC3D7 [11,12]. Only size variation, due to the different number of repeats, has been observed within the glurp gene [9].

The present analysis was conducted as part of a clinical trial of artesunate (AS) monotherapy designed to probe for and characterize clinical artemisinin resistance in adult patients with uncomplicated P. falciparum malaria. The trial was conducted in a rural district of Western Cambodia, close to the international border with Thailand and where reports of AS treatment failures had already emerged [13]. The main objective of this study was to describe changes in parasite genotype during seven days of AS treatment in patients classified as artemisinin resistant and assess for emergence of novel (minority) parasite populations that were not detected at baseline. Secondary objectives were: (1) to describe the diversity of msp1, msp2 and glurp in this Cambodian study population, and (2) to assess whether conventional classification of recrudescence/re-infection based on samples collected only at baseline and day of failure $\left(D_{f}\right)$ remained valid once interim sampling was performed. There were two working hypotheses. Firstly, that AS-resistant patients were infected with novel subpopulations of relatively drug-resistant parasites not identified at baseline; as the majority of more sensitive parasites were killed, the resistant parasites would persist and become detectable during a seven-day course of AS therapy. Secondly, that new falciparum infections during follow-up had persistence of these intrinsically more resistant parasite subpopulations. Hence, these cases should be reclassified as recrudescent, making the true failure rate higher with longitudinal genotyping compared to the conventional time points of baseline and $\mathrm{D}_{\mathrm{f}}$.

\section{Methods}

\section{Clinical methods}

\section{Study setting and participants}

The study was part of a randomized clinical trial conducted during 2008-2009 at Tasanh Health Center located in Battambang Province in Western Cambodia, a region of low malaria transmission, with just one third 
of the study population reporting one or more previous episodes of malaria. Full details of the study are described elsewhere [14]. Briefly, after giving informed consent, screening procedures were conducted, and eligible patients with uncomplicated $P$. falciparum malaria were randomized to receive one of three AS monotherapy regimens given as a single daily oral dose: 2 , 4 or $6 \mathrm{mg} / \mathrm{kg} /$ day ( $\mathrm{n}=75,40$, and 28 , respectively) for seven days. Inclusion criteria were age 18 to 65 years, fever or history of fever within 48 hours of presentation, monoinfection with $P$. falciparum as determined on Giemsastained thick and thin blood films by light microscopy, and parasite density 1,000-200,000 asexual parasites/ml blood. Exclusion criteria were signs and symptoms of severe malaria and documented use of anti-malarial drugs within the preceding 30 days. All patients remained on the study ward for the first week until completion of AS administration and returned for weekly outpatient review until Day 42. Patient outcomes for the main treatment study were classified according to clinical and parasitological responses [15]. Primary analysis for this molecular study was intent-to-treat so as to include additional subjects who had given informed consent to participate, including withdrawal and screening failure cases where a baseline blood sample for molecular genotyping had been collected. The study was approved by the WRAIR IRB, World Health Organization Ethical Review Committee (WHO ERC), and the Cambodian Ethics Committee for Human Research (NECHR).

For the longitudinal genotyping analysis conducted over the first seven days (Days 0 to 6), three subgroups of patients were selected and defined as follows: (Group A) "artemisinin sensitive" (rapid resolution of symptoms plus asexual parasite clearance time $<48$ hours by microscopy and remained cured to 42 days) $(\mathrm{n}=18)$; (Group B) "slow parasite clearance" (parasite clearance time $>96$ hours by microscopy and remained cured of P. falciparum throughout the follow up period $(\mathrm{n}=14)$; (Group $\mathrm{C})$ "Recurrence" (re-appearance of asexual P. falciparum parasites, as determined by light microscopy, during follow up after initially clearing parasitaemia, with or without clinical symptoms/ signs of malaria $(n=9)$. Groups $B$ and $C$ when combined were termed "artemisinin resistant". Group D constituted the remaining patients not included in A, B or C. For the purposes of the present analysis, enrolled patients developing Plasmodium vivax mono-infection during followup and confirmed by vivax-specific PCR were considered cured of $P$. falciparum infection.

\section{Sample collection}

Prior to initiation of AS therapy (Day 0), blood for parasite density, species confirmation and genotyping was collected into EDTA. Further samples were collected daily on Days 1 to 6 and again on the day of treatment failure $\left(D_{f}\right)$ if malaria recurrence occurred. All samples were transferred to cryovials, stored in liquid nitrogen at the study site for transfer to Bangkok, and then kept frozen at $-80^{\circ} \mathrm{C}$ until used for DNA extraction.

\section{Laboratory methods \\ Genotyping of Plasmodium falciparum}

Genomic DNA was extracted from $200 \mu \mathrm{l}$ whole blood per patient using the Qiagen DNA extraction kit (QIAGEN, USA) according to manufacturer's instructions. The polymorphic regions of the merozoite surface proteins msp1 (block 2), msp2 (block 3), and glurp (R2 repeat region) were amplified by nested PCR. In the primary reaction, the oligonucleotide primers corresponded to conserved sequences within $m s p 1$ (block 2), $m s p 2$ (block 3 ) and glurp, and in the nested reaction, separate primer pairs targeted the respective allelic types of msp1 (K1, MAD20, and RO33) and $m s p 2$ (FC27 and IC3D7) for amplification [11]. For controls, genomic DNA from a 3D7 laboratory strain was isolated by standard techniques. Positive and negative controls were systematically incorporated in each PCR run. The msp1, msp 2 and glurp PCR products were loaded on $2 \%$ agarose gels, stained with ethidium bromide, separated by electrophoresis and visualized under UV trans-illumination (VersaDoc ${ }^{\circ}$, BIORAD, Hercules, USA). Samples from an individual patient were run in adjacent lanes. If there was no amplification for any allelic family, the PCR was repeated with three times the quantity of template DNA. If no amplification was detected after this second reaction, amplification was classified as unsuccessful. Analyses of number of genotypes and size polymorphism were digitalized using Quantity $\mathrm{One}^{\circ}$ software (Bio-Rad Laboratories, Hercules, USA). A minimum of ten base pairs size difference was required to define an additional genotype.

\section{Determination of PCR-corrected response}

Comparison of genotyping patterns of $m s p 1, m s p 2$ and glurp at baseline and at $\mathrm{D}_{\mathrm{f}}$ was used to classify parasitological outcomes for LTF cases. Genotyping was done stepwise with an initial $m s p 2$ determination, followed by msp1 genotyping of the paired blood samples found to have at least one identical $m s p 2$ allele before and after treatment. Based on analysis of the 3D7 strain, alleles were considered the same if molecular weights were within ten base pairs (bp) [2,6]. It was assumed that after a patient was initially treated for malaria, a subsequent malaria episode was caused by either parasite strains present before treatment (e.g., recrudescence) or parasite strains acquired after completion of treatment (e.g., new infection). An outcome was defined as recrudescence if a subsequent sample contained identical alleles or a subset of the alleles present in the first sample [8]. If a subsequent sample contained alleles present in the first 
sample and new alleles, the outcome was still considered recrudescent. An outcome was defined as a new infection if a subsequent sample contained only new alleles.

Allelic variants were analysed only with respect to the size of polymorphisms given the high level of discrimination afforded by the Quantity One ${ }^{\circ}$ software computer analysis.

\section{Statistical methods}

Continuous data were expressed as medians with interquartile ranges or, in the case of parasite counts, as geometric means with $95 \%$ confidence intervals $(95 \% \mathrm{CI})$. Continuous data from patient groups were compared using the non-parametric Kruskall-Wallis test. Numerical data were expressed as proportions and compared using Chi-square or Fisher's exact tests as appropriate. All statistical tests were performed at the $5 \%$ significance level and corresponding 95\% confidence intervals were estimated. Statistical analyses were performed using SPSS version 12.0 (SPSS Inc., Chicago, IL, USA) and Stata version 11 (College Station, TX, USA).

\section{Results}

PCR genotyping was successful for one or more genes in baseline samples from 143 patients who had undergone screening procedures prior to study enrolment ( $m s p 1$ 125, msp2 137 and glurp 143). Four patients were subsequently determined not to meet all treatment study entry criteria, but genotype samples from these cases were retained for this molecular analysis. Some 139 patients were randomized to one of three oral AS monotherapy regimens; three were subsequently lost to follow-up while the remaining 136 were followed until a study endpoint was met. One case represented with recurrence 56 days after enrolment into the treatment study and was included in this analysis; another developed neutropenia on D3 and was withdrawn from the treatment study but retained for safety follow up and developed malaria recurrence at D42. Baseline characteristics and clinical/parasitological outcomes for patients are given in Table 1. Subjects termed artemisinin-resistant (Groups B and $\mathrm{C}$ combined) had significantly higher presenting parasitaemia and slower parasite clearance rates and times than those in Group A (artemisinin-sensitive) ( $<0.0001$ for all comparisons). Approximately one quarter of the study population, including nearly half of the malaria recurrence cases, had gametocytes documented by microscopy at some point during the 42-day study follow-up period; however, gametocytes were detected in only two cases at the same time a PCR genotyping sample was collected: one in Group A (at baseline) and one in Group C (at $\mathrm{D}_{\mathrm{f}}$ ).

\section{Population diversity of msp1, msp2 and glurp at baseline Population diversity}

There was more genotypic variation in $m s p 2$ (51 alleles) than msp1 (24 alleles) and glurp (34 alleles) (Figure 1).
The length variations of the $m s p 1$-amplified product were approximately 100-330 bp for K1, 150-320 bp for MAD20 and 220-230 bp for RO33. For msp2, the length variations of the amplified product were approximately 170-820 bp for FC27 and 290-870 for IC3D7, while glurp showed 660-1,090 bp of length variation.

\section{Haplotypes}

Some 13 distinct patterns of genotype (haplotypes) were observed at baseline (Tables 2 and 3, Figure 2). Recurrent cases were comprised of just three haplotypes: msp1-K1/ msp2-IC3D7, msp1-K1/msp2-FC27 and msp1-MAD20/ msp2-FC27; these three haplotypes were also prominent in Groups A and B, and therefore probably not associated with clinical phenotype. In contrast, haplotypes containing msp2-RO33 were observed in some AS-sensitive cases $(5 / 18,28 \%)$, but not in the AS-resistant subgroup.

\section{Complexity of infection}

Overall, 45/143 (31\%) of the study population had evidence of a polyclonal infection at baseline with two or more $m s p 1, m s p 2$ or glurp alleles present (Tables 2 and 3). Multiple alleles were detected in 17\% (21/125) msp1, 26\% (36/138) msp2 and 8\% (12/143) glurp samples, respectively. The maximum number of clones seen for a single gene was six (one case with six clear bands of $m s p 2-\mathrm{FC} 27$ in addition to two clones of $m s p 1-\mathrm{K} 1$ ); this case remained cured at 42 days but had a prolonged parasite clearance time of 102 hours. All other polyclonal infections had just two or three clones. In the three subgroups of artemisinin sensitive, slow clearers and recurrences, multiple P. falciparum alleles of msp-1, msp-2 and glurp, were detected in 5/18, 3/14 and 7/9 patients $(28,21$ and $79 \%$, respectively) (Figure 3). Recurrence patients were significantly more likely to have polyclonality of one or more alleles demonstrated at baseline than patients without falciparum recurrence (seven of nine versus 36 of 127, $\mathrm{p}=0.004$ ). Five of nine cases were polyclonal at $\mathrm{D}_{\mathrm{f}}$.

\section{Parasite genotype during seven-day artesunate therapy and at failure}

Parasite genotype was evaluated daily on Days 0-6 of AS therapy for individual recurrence cases (Figure 3). In all participants, peripheral parasitaemia cleared by Day 4 of AS therapy although genotyping PCR assay performed retrospectively demonstrated the continued presence of parasites through Day 6.

\section{Detection of minority parasite populations during treatment}

Only one case of recurrence had a new allele (msp23D7) observed on Days 3 and 5, when asexual parasitaemia was still positive by light microscopy; this could 
Table 1 Baseline characteristics and clinical and parasitological outcomes in groups of patients with Plasmodium falciparum malaria followed with sequential parasite genotyping during 7 days of artesunate monotherapy

\begin{tabular}{|c|c|c|c|c|c|c|}
\hline & $\begin{array}{c}\text { Group A } \\
\text { Artemisinin } \\
\text { "sensitive" }\end{array}$ & $\begin{array}{c}\text { Group B } \\
\text { Slow parasite } \\
\text { clearance }\end{array}$ & $\begin{array}{c}\text { Group C } \\
\text { Recurrence }\end{array}$ & $\begin{array}{c}\text { Groups B + C } \\
\text { Artemisinin } \\
\text { "resistant" }\end{array}$ & $\begin{array}{c}\text { Group D } \\
\text { Remaining } \\
\text { study cases }\end{array}$ & $\begin{array}{l}\text { Overall study } \\
\text { population }\end{array}$ \\
\hline Number of cases & 18 & 14 & 9 & 23 & 102 & 143 \\
\hline Age (y), median (IQR) & $25(18-32)$ & $25(21-31)$ & $22(18-27)$ & $23(20-31)$ & $25(20-38)$ & $25(20-35)$ \\
\hline Male:female, n (\%) & $16: 2(89: 11)$ & 13:1 (93:7) & $6: 3(67: 33)$ & 19:4 (83:17) & $77: 25(75: 25)$ & 112:31 (78:22) \\
\hline Parasitaemia (/uL), geometric mean $(95 \% \mathrm{Cl})$ & $6,698(3,564-12,589)$ & $51,944(28,540-94,542)$ & $22,209(9,193-53,653)$ & $37,251(22,776-60,926)$ & $11,527(4,933-32,687)$ & $14,207(4,891-37,778)$ \\
\hline Allocated daily AS monotherapy dose: 2 - 4 - 6 (mg/kg/day), $\mathrm{n}^{+}$ & $10-5-3$ & $3-8-3$ & $4-2-3$ & $7-10-6$ & $58-25-19$ & $71-40-28$ \\
\hline Parasite clearance time by microscopy (h), median (IQR) & $36(30-42)$ & $108(102-108)$ & $96(84-96)$ & $102(96-108)$ & $74(66-84)$ & $78(60-90)$ \\
\hline Parasite clearance rate* $(/ \mathrm{h})$, median (IQR) & $0.086(0.059-0.11)$ & $0.036(0.033-0.038)$ & $0.035(0.030-0.041)$ & $0.036(0.033-0.039)$ & $0.040(0.034-0.047)$ & $0.040(0.034-0.052)$ \\
\hline \multicolumn{7}{|l|}{ Parasitological outcome at D42, n (\%) } \\
\hline Cured & $18(100)$ & $14(100)$ & $1(11)^{\wedge}$ & $15(65)^{\wedge}$ & $96(96)$ & $131(92)$ \\
\hline Recrudescence** & 0 & 0 & $7(67)$ & $7(30)$ & $0(0)$ & $7(5)$ \\
\hline New Pf infection** & 0 & 0 & $1(11)$ & $1(4)$ & $0(0)$ & $1(1)$ \\
\hline No outcome recorded & 0 & 0 & 0 & 0 & $4(4)^{+}$ & $4(3)$ \\
\hline \multicolumn{7}{|l|}{ Gametocytes by microscopy, n (\%) } \\
\hline At baseline & $1(6)$ & $1(7)$ & $1(11)$ & $2(9)$ & $16(16)$ & $19(13)$ \\
\hline During week 1 & $3(17)$ & $1(7)$ & $1(11)$ & $1(4)$ & $26(25)$ & $30(21)$ \\
\hline Any time D0-42 & $3(17)$ & 0 & $4(44)$ & $4(17)$ & $29(28)$ & $36(26)$ \\
\hline
\end{tabular}

Key. * Calculated using slope of curve for $\log _{10}$-normalised parasite clearance as determined by light microscopy [14].

${ }^{+} 4$ screening failure cases received standard AS-MQ treatment and had baseline data recorded only.

** Outcome determined using genotyping based on $m s p 1, m s p 2$ and glurp fragment sizes on paired blood samples collected at baseline and $D_{f}$

$\wedge$ Includes one case classified per protocol as cured on D42 but self-presented with recurrent malaria on D58 which was subsequently determined to be recrudescence by PCR genotyping.

$\mathrm{h}=$ hours; $\mathrm{y}=$ years.

Significant differences exist between artemisinin sensitive and resistant groups for parasitemia, parasite clearance time and parasite clearance rate ( $p=0.0001$ for all comparisons). 


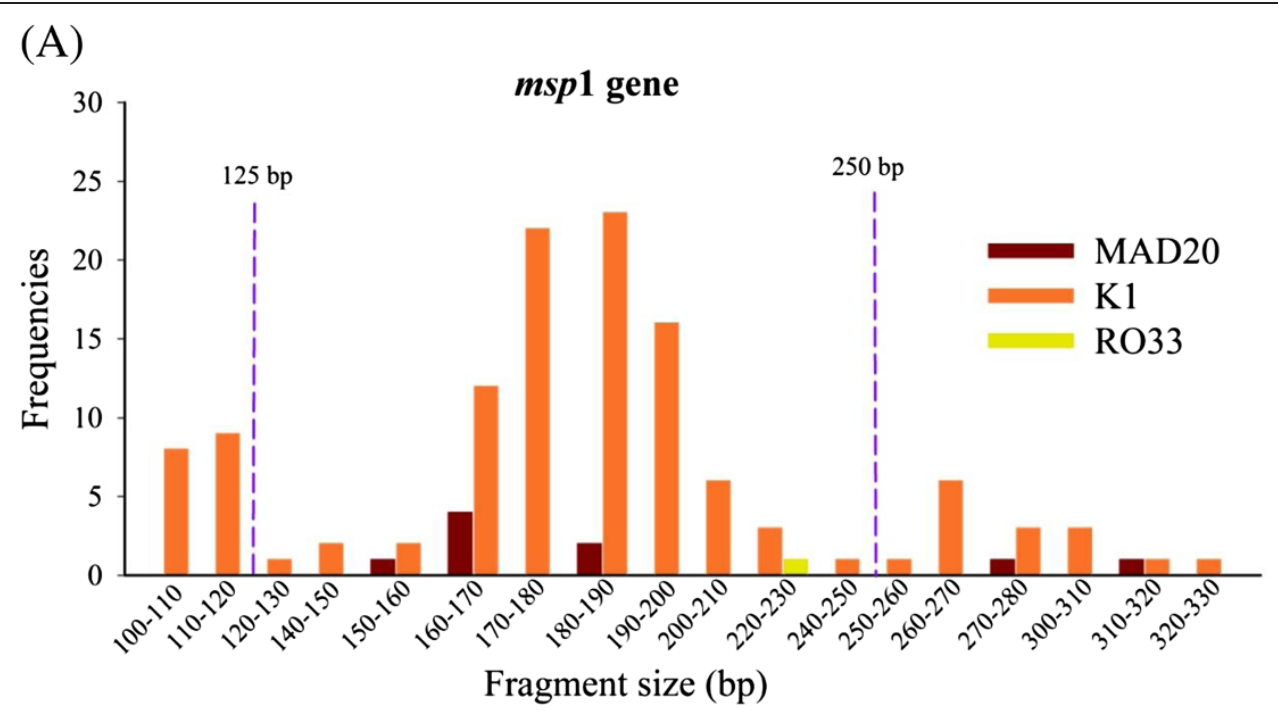

(B)

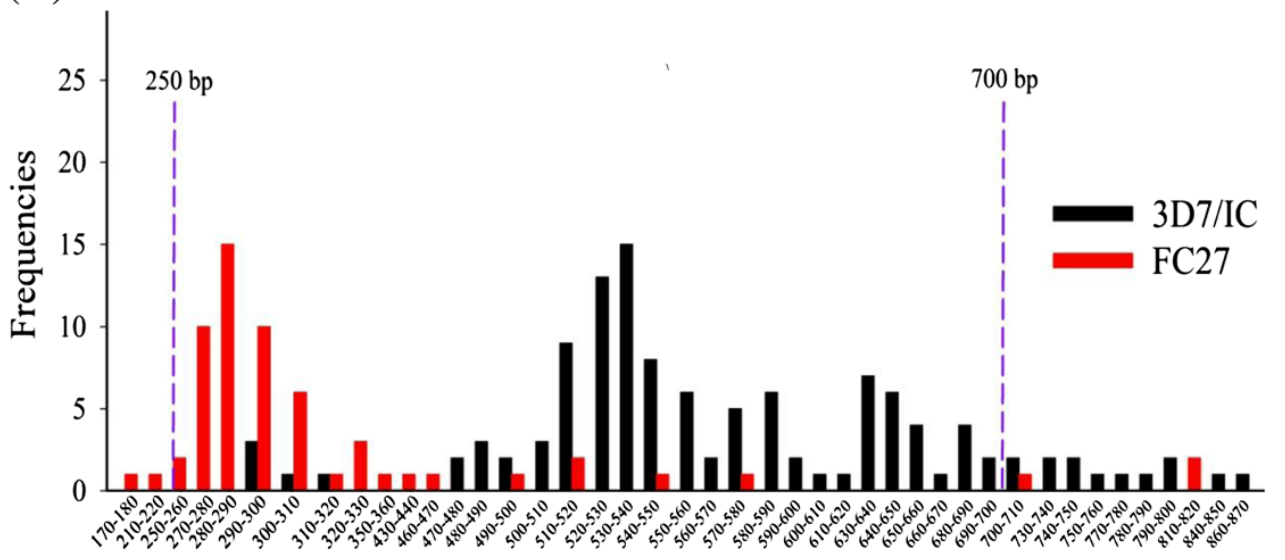

Fragment size (bp)

(C)

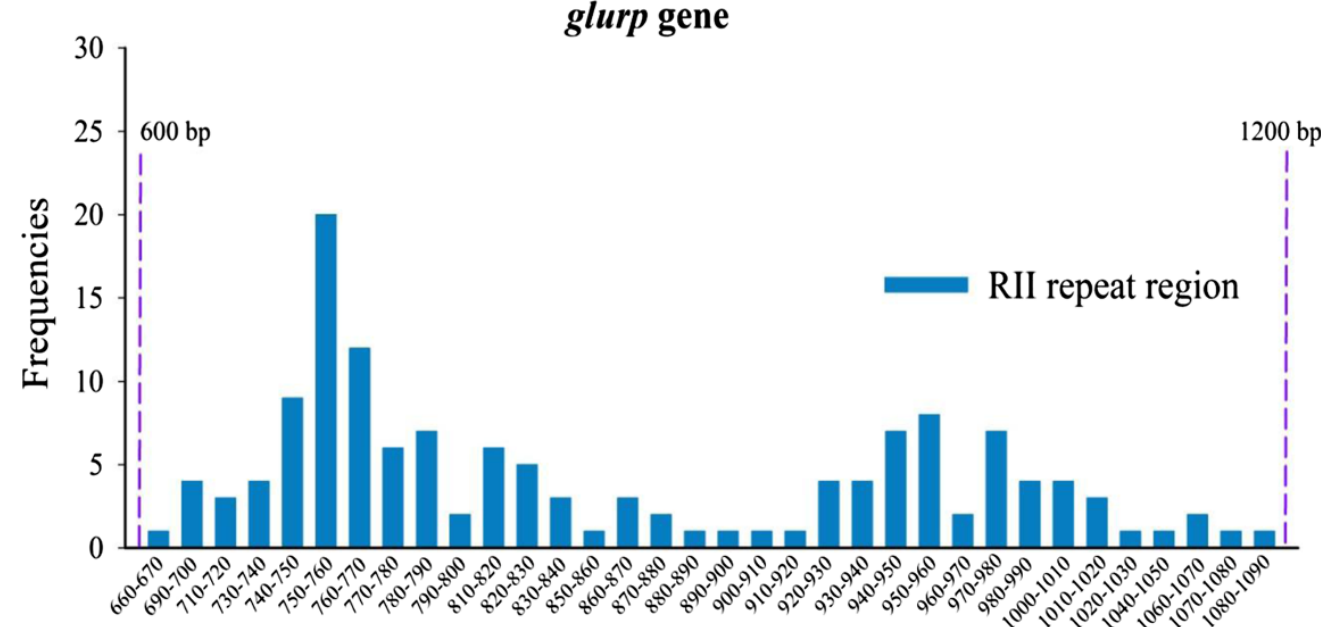

Fragment size (bp)

Figure 1 (See legend on next page.) 
(See figure on previous page.)

Figure 1 Parasite genetic diversity detected by $m s p 1, m s p 2$ and glurp. Polymerase chain reaction (PCR) products were categorized into molecular weight groups differing by 10 bp. (A) msp1. Orange bars represent K1; brown bars, MAD-20; and yellow bars, RO33 allelic families. The $18 \mathrm{~K} 1$ alleles ranged in size from 100 to $330 \mathrm{bp}$; the 5 MAD-20 alleles; 150 to 320 bp; and the 1 RO33 alleles, 220-230 bp. (B) msp2. Red bars represent FC27 and black bars represent IC3D7 allelic families. The 18 FC27 alleles ranged in size from 170 to 820 bp, and the 33 IC3D7 alleles ranged in size from 290 to 870 bp. (C) glurp. The glurp alleles ranged in size from 660 to 1,090 bp. Dashed purple lines represent fragment size thresholds for each gene [10].

represent a minority clone potentially relatively resistant to AS treatment. However, this allele was not detected again at $D_{f}$. In the remaining eight recurrence cases, while fewer of the alleles present at baseline were detected as parasitaemia cleared, no new alleles emerged.

In the 14 patients with prolonged parasite clearance but no recurrence of malaria (Group B) no minority alleles emerged during seven days of treatment.

\section{Determination of parasitological outcome in recurrent malaria (LTF) cases}

Based on conventional genotyping methodology using only baseline and $D_{f}$ samples, eight of nine (89\%) Plasmodium falciparum recurrences were determined to be recrudescent and one of nine (11\%) was a new infection. Longitudinal genotyping on days 1-6 revealed that in six of nine cases no new minority parasite populations were

Table 2 Haplotypes of $m s p 1$ and $m s p 2$ seen in 143 cases of uncomplicated $P$. falciparum malaria

\begin{tabular}{|c|c|c|c|c|c|c|c|c|}
\hline \multirow[t]{3}{*}{ Type } & \multicolumn{2}{|c|}{ Haplotype pattern } & \multirow{3}{*}{$\begin{array}{l}\text { Polyclonal } \\
\text { for }>1 \\
\text { allele }\end{array}$} & \multicolumn{5}{|c|}{ Number of cases } \\
\hline & msp1 & msp2 & & Fast clearers & Slow clearers & Malaria recurrences & Remaining cases & Total population \\
\hline & & & & $(n=18)$ & $(n=14)$ & $(n=9)$ & $(n=102)$ & $(n=143)$ \\
\hline \multirow[t]{2}{*}{1} & MAD20 & IC3D7 & No & 1 & - & - & 1 & 2 \\
\hline & & & Yes & - & - & - & 1 & 1 \\
\hline \multirow[t]{2}{*}{2} & MAD20 & $\mathrm{FC} 27$ & No & - & - & - & 1 & 1 \\
\hline & & & Yes & - & - & - & - & - \\
\hline \multirow[t]{2}{*}{3} & MAD20 & IC3D7/FC27 & No & - & - & - & - & - \\
\hline & & & Yes & 1 & - & - & - & 1 \\
\hline \multirow[t]{2}{*}{4} & MAD20/K1 & IC3D7/FC27 & No & - & - & - & 1 & 1 \\
\hline & & & Yes & - & 1 & - & - & 1 \\
\hline \multirow[t]{2}{*}{5} & K1 & IC3D7 & No & 3 & 4 & - & 35 & 42 \\
\hline & & & Yes & 3 & - & 7 & 13 & 23 \\
\hline \multirow[t]{2}{*}{6} & K1 & $\mathrm{FC} 27$ & No & 3 & 5 & 1 & 17 & 26 \\
\hline & & & Yes & - & - & - & 6 & 6 \\
\hline \multirow[t]{2}{*}{7} & K1 & IC3D7/FC27 & No & 1 & 1 & - & 3 & 5 \\
\hline & & & Yes & 1 & 2 & - & 5 & 8 \\
\hline \multirow[t]{2}{*}{8} & K1 & - & No & - & - & - & 4 & 4 \\
\hline & & & Yes & - & - & - & 2 & 2 \\
\hline \multirow[t]{2}{*}{9} & $\mathrm{RO} 33$ & IC3D7 & No & 2 & - & - & - & 2 \\
\hline & & & Yes & - & - & - & - & - \\
\hline \multirow[t]{2}{*}{10} & $\mathrm{RO} 33$ & FC27 & No & 2 & - & - & - & 2 \\
\hline & & & Yes & - & - & - & - & - \\
\hline \multirow[t]{2}{*}{11} & RO33 & IC3D7/FC27 & No & 1 & - & - & 1 & 2 \\
\hline & & & Yes & - & - & - & 1 & 1 \\
\hline \multirow[t]{2}{*}{12} & - & IC3D7 & No & - & - & - & 6 & 6 \\
\hline & & & Yes & - & - & - & 2 & 2 \\
\hline \multirow[t]{2}{*}{13} & - & FC27 & No & - & 1 & 1 & 3 & 5 \\
\hline & & & Yes & - & - & - & - & - \\
\hline
\end{tabular}


Table 3 Number of patients with msp1 or msp2 genes detected at baseline

\begin{tabular}{|c|c|c|c|c|c|}
\hline Genotype & $\begin{array}{l}\text { Artemisinin sensitive } \\
\qquad n=18\end{array}$ & $\begin{array}{l}\text { Slow parasite clearers } \\
\qquad n=14\end{array}$ & $\begin{array}{c}\text { Recurrences } \\
n=9\end{array}$ & $\begin{array}{l}\text { Remaining cases } \\
\qquad n=102\end{array}$ & $\begin{array}{c}\text { Total population } \\
n=143\end{array}$ \\
\hline \multicolumn{6}{|l|}{ msp1 } \\
\hline MAD20 & $2(29)$ & $1(14)$ & 0 & $4(57)$ & 7 \\
\hline K1 & $11(9)$ & $13(11)$ & $8(7)$ & $86(73)$ & 118 \\
\hline $\mathrm{RO} 33$ & $5(72)$ & 0 & 0 & $2(29)$ & 7 \\
\hline No msp 1 detected & 0 & $1(8)$ & $1(8)$ & $11(85)$ & 13 \\
\hline \multicolumn{6}{|l|}{ msp2 } \\
\hline IC3D7 & $13(14)$ & $8(8)$ & $7(7)$ & $69(71)$ & 97 \\
\hline $\mathrm{FC} 27$ & $9(15)$ & $10(17)$ & $2(3)$ & $38(65)$ & 59 \\
\hline No msp2 detected & 0 & 0 & 0 & $6(100)$ & 6 \\
\hline
\end{tabular}

detected during treatment, thus supporting the classification of recrudescence, although not all alleles present at baseline were detected at the time of recurrence. In one case (participant 140) described above, minority alleles were observed on Days 3 and 5 of AS treatment but not on $\mathrm{D}_{\mathrm{f}}$. and thus the classification remained as recrudescence. One case (88) was also classified as recrudescent based on the appearance of at least one allele present in the baseline sample, as well as novel alleles at $\mathrm{D}_{\mathrm{f}}$. Case 112 was classified as a new infection based on the appearance of novel alleles at the time of recurrence, none of which were observed during the first week. Thus in all nine cases of malaria recurrence, additional genotyping during AS treatment on days 1-6 did not alter classification of the final parasitological outcome.

\section{Discussion}

This study aimed to detect emerging drug-resistant minority parasite clones during slowly clearing malaria infections that could account for cases of unrecognized treatment failure in a setting of clinical AS resistance. In fact, of the 23 cases in the artemisinin-resistant subgroup in whom daily parasite genotyping was undertaken for seven days, including nine cases with recurrent disease, a minority clone was detected in only one; moreover this clone was not present at $\mathrm{D}_{\mathrm{f}}$, so did not appear to be associated with subsequent treatment failure. Two of the 23 cases had novel alleles present at $D_{f}$ but these had not been detected at all during AS therapy despite the sensitivity of the molecular methods used. In this Cambodian setting, where most enrolled patients
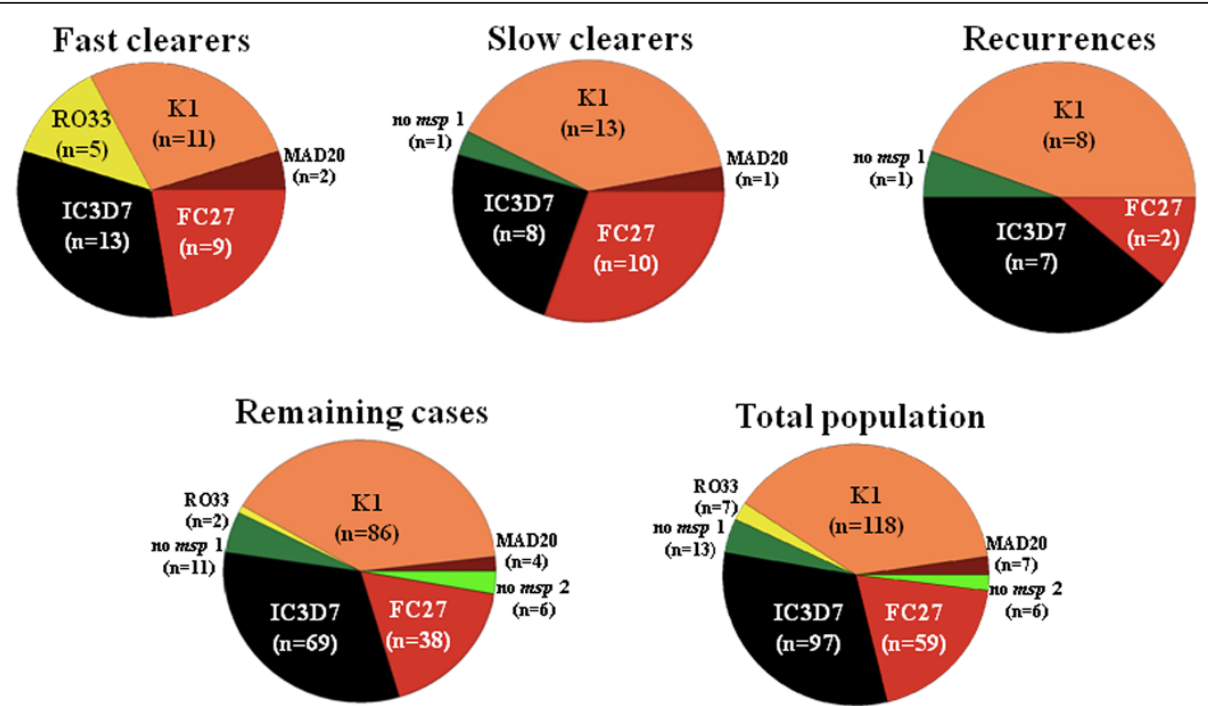

Figure 2 Pie charts showing genotype frequencies of $m s p 1$ and $m s p 2$ determined at baseline for fast parasite clearers (artemisinin sensitive) $(n=14)$, slow parasite clearers $(n=18)$, recurrences $(n=9)$, and for remaining $(n=102)$ and overall $(n=143)$ study populations. Key: orange, brown, yellow = msp 1; dark green = no $m s p 1$; black, red = $m s p 2$; lime green = no $m s p 2$. 


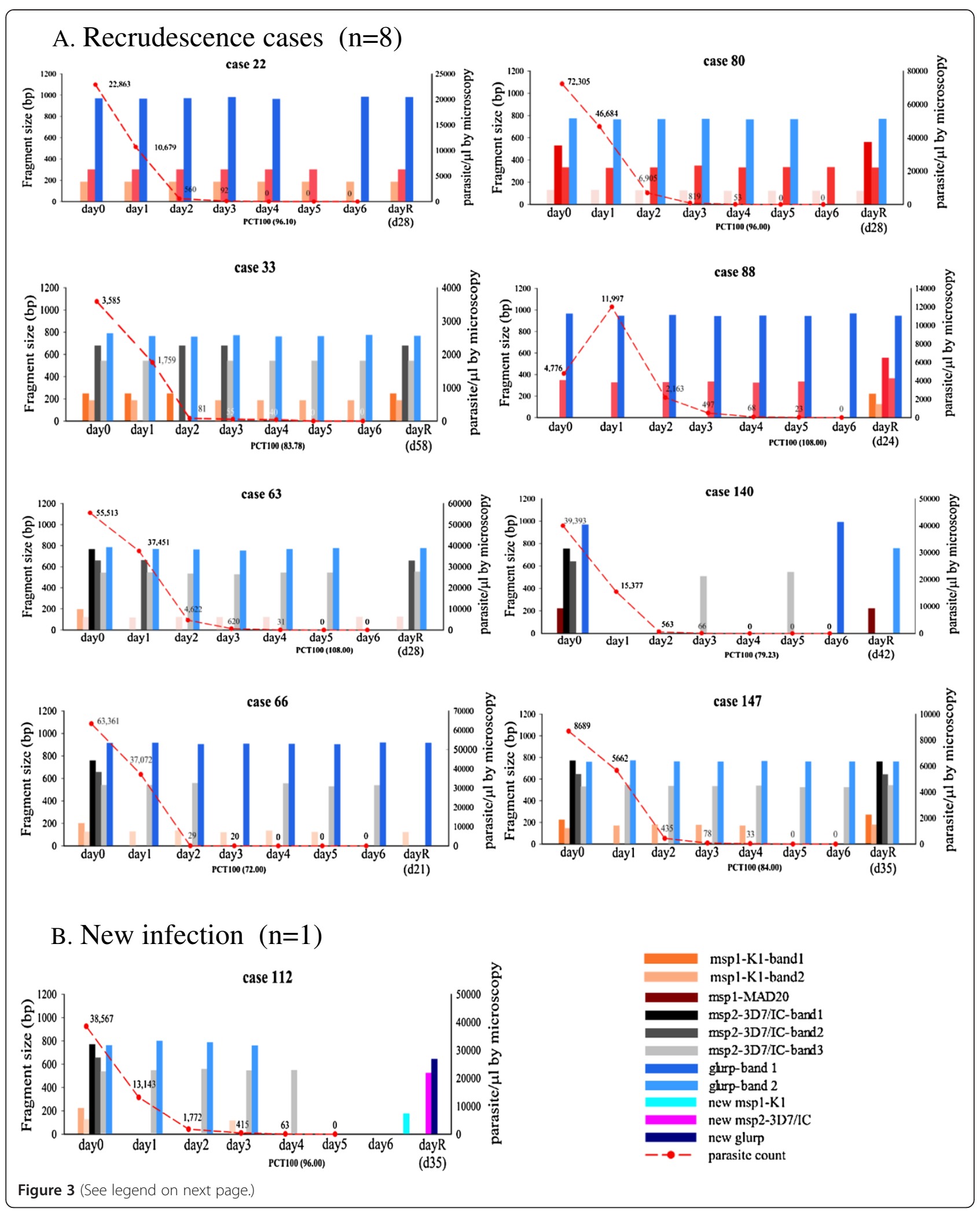


(See figure on previous page.)

Figure 3 Parasite genotype determined daily in individual patients with malaria recurrence (Group C). Allele typing displaying size variation of $18 \mathrm{~s}$ alleles for $\mathrm{msp} 1, \mathrm{msp} 2$ and glurp in P. falciparum parasites isolated from nine patients showing the patterns of alleles present during seven days of AS monotherapy and on the day of falciparum malaria recurrence. Key: $\mathrm{BP}=$ base pairs; PCT100 = duration of parasite clearance by microscopy; bands = separate clones for the same allele; dayR = day of malaria recurrence. Dashed lines represent asexual parasitaemia determined by microscopy. (A) Recrudescence cases. Case 66 received only three days of initial AS treatment due to development of neutropenia and completed anti-malarial therapy with an alternative short-acting drug. All remaining cases completed seven days of AS monotherapy. Gametocytes were seen by microscopy for cases 33 (Day 0-21), 80 (Days 35 and 42), and 147 (Days 21 and 42 ). Case 88 had two shared alleles on $D_{f}$ plus additional msp1 and msp2 loci. Case 140 had one new allele of msp2 detected on Days 3 and 5 but not on Day 0 or $D_{f}$ One shared msp1 allele detected at $D_{f}$ plus 1 new allele of glurp. (B) New infection. Case 112 new alleles of msp1, msp2 and glurp detected on $D_{f}$ in addition to Plasmodium vivax.

had persistence of asexual parasitaemia beyond 72 hours, the parasites responsible for slow parasite clearance and recurrent malaria are the dominant or co-dominant clones in an acute infection. Further, because they are also present in fast parasite clearers, they are well established in the Tasanh parasite population. These data also support recent reports of highly conserved, yet genotypically very distinct, populations of parasites from a number of sites in Cambodia including this study site [4].

In this setting of low malaria transmission and emerging drug resistance, considerable heterogeneity within the P. falciparum parasites was revealed in baseline samples from the symptomatic patient population. In keeping with surveys from West Africa and Thailand, these Cambodian isolates showed greater polymorphism of the $m s p 2$ allelic family (IC3D7 and FC27) compared to $m s p 1$ and glurp $[12,16]$. These results showing the distribution and diversity of $m s p 1, m s p 2$ and glurp in a relatively isolated area of Western Cambodia are similar to those from directly across the border in Trat Province, Thailand [12]. Comparative data from other locations in Cambodia is currently lacking.

Evidence of polyclonal infection, in the form of the presence of two or more alleles from msp1, $m s p 2$ and glurp within a single sample, was found in just $31 \%$ of subjects overall at baseline (17, 26 and $8 \%$ for $m s p 1$, msp 2 and glurp, respectively). This suggests a relatively low diversity of the $P$. falciparum parasite population in this localized area of Western Cambodia. A study from Kampala, Uganda, also a relatively low transmission area, found $45 / 98$ (46\%) samples were polyclonal for msp 2 at baseline [17], while in Kenya $60 \%$ of asymptomatic blood smear positive children in an area of moderate transmission in Kenya had two or more $m s p 2$ clones detected [18]. For $m s p 132 \%$ of 45 symptomatic and $64 \%$ of 45 asymptomatic infections in a hyperendemic region of Gabon had polyclonality of $m s p 1$ [19]. An intriguing finding in this Cambodian study was that patients who subsequently developed recurrent disease were significantly more likely to harbour two or more clones at baseline compared with the rest of the study population. Since this is a location in which slow parasite clearance during AS treatment is the norm rather than the exception, the majority of slow clearers also had monoclonal infections; this raises a question as to whether, in addition to simply defining resistance as a certain proportion of cases with prolonged parasite clearance time or half-life, we should be broadening current research objectives to include identification and follow-up of recurrent polyclonal infections so as to define their role as potential agents of emerging resistance.

In the present study, six clones were demonstrated in one baseline sample, although only two or three clones were detected in the remaining 44 patients with polyclonal infections. In general, studies from high transmission areas have shown that asymptomatic malaria patients harbour more parasite clones than those with symptomatic disease [20]. The same is true for asymptomatic versus severe malaria: in a post-mortem study from Malawi, tissue genotyping for sequestered parasites demonstrated that children with cerebral malaria had sequestered parasite populations less genetically complex than both their circulating parasites and the circulating parasites in an asymptomatic malaria group, despite the much higher parasite burden [21]. The more homogeneous distribution of genotypes in cerebral malaria than in asymptomatic cases suggested the emergence of a single or small number of more dominant parasite clones, although the authors did acknowledge that the sheer burden of parasites in cases of cerebral malaria may have prevented lesser populations from being detected by PCR. Likewise, in this present study, conducted in a localized low transmission setting in Western Cambodia, cases of symptomatic disease, including most cases with very prolonged parasite clearance, tended to be dominated by single parasite clones. However, while there appeared to be an association of RO33 with some AS sensitive cases, no haplotype could be identified to be indicative of a resistant phenotype. Newer techniques, such as the heteroduplex tracking assay $[22,23]$, may yield greater discrimination of clonal populations and identify haplotypes other than those used in this present analysis.

Additional daily samples were genotyped during seven days of AS treatment (Days 0 to 6) as well as on the day 
of recurrent $P$. falciparum parasitaemia $\left(D_{\mathrm{f}}\right)$, which occurred between 21 and 58 days after commencing AS. Genotyping patterns from seven of nine treatment failure cases showed multiple parasite alleles of $m s p 1$ and/ or $m s p 2$ at baseline, although only a minority were then detected during the following days of AS treatment. This may be due to greater sensitivity of some parasite strains to AS treatment, as well as to declining parasitaemia in the small volume of sampled blood, or to a combination of both. An alternative explanation for the disappearance of some parasite strains, which later re-appear at the time of treatment failure, is parasite dormancy triggered by the presence of AS $[24,25]$.

However, repeated genotyping of symptomatic travellers returning from Africa before and during 96 hours of quinine treatment for $P$. falciparum malaria in France revealed 19/20 (95\%) cases harboured between two and five clones, with some cases having up to three dominant clones in addition to other minority clones [26]. Moreover, quantification of clones revealed that while the parasitaemia of some declined steadily during drug treatment, others showed major fluctuations of parasitaemia that were apparently unrelated to the presence of drug. Thus, a single baseline blood sample may provide incomplete information on the diversity of parasite populations present within a given patient. In contrast, Färnert and colleagues found only one of 13 patients with symptomatic malaria had new clones detected sporadically during 12 -hourly sampling over a three to nineday period, despite more than half (seven of 13) having multiple clones of $m s p 1$ and $m s p 2$ detected at baseline [27]. This latter study reflects the findings in this study in which just one of nine treatment failures and none of 14 slow parasite clearers had new alleles detected during treatment.

A striking feature in patients undergoing longitudinal sampling over seven days was the presence of parasite DNA up to seven days after the initiation of AS therapy; this may represent relatively drug-resistant $P$. falciparum populations undergoing selection or modification in the presence of drug. Parasite DNA is detectable in peripheral blood for less than 48 hours following the death of a parasite in response to drug or immune mechanisms and before being removed from the circulation by circulating phagocytes and spleen macrophages [28]. Therefore the presence of parasite DNA in blood beyond the microscopy-calculated parasite clearance time does likely represent the persistence of viable parasites. As well as the increased sensitivity of PCR compared to microscopy (in general 0.1 versus 10 parasites/ $\mu \mathrm{L}$ [29]), an additional explanation for the persistently positive PCR results up to the final day of AS treatment is the presence of gametocytes in the blood samples. However, in this study only one of 23 patients in the AS-resistant group had gametocytes detected by microscopy during the first seven days, and just one of nine LTF cases had gametocytes on $\mathrm{D}_{\mathrm{f}}$. Therefore the quantification results are unlikely to be influenced significantly by the presence of circulating gametocytes. Using current PCR strategies, gametocytes can only be detected differentially by reverse transcription-PCR (RT-PCR) [30]. The ability to differentiate gametocytes from asexual parasites would enable a more reliable estimate of true failure rates and thus support efforts to limit the spread of drug resistance and meet containment and elimination targets.

In this study, conventional genotyping assigned the outcome of six of nine samples as recrudescence; additional genotyping on Days 1-6 did not alter this assessment. In two further cases, while the final classification was not altered by additional genotyping the presence of additional bands does illustrate some of the dilemmas inherent in this genotyping technique. Mixed genotype results may profoundly impact estimates of drug resistance if they form a significant proportion of treatment failure cases in clinical trials, and depending on how they are interpreted [8]. If circulating strains at low parasite densities are not detected in baseline samples because other (dominant) parasite strain(s) are present in much larger quantities, they could still emerge later to cause treatment failure, leading to the misclassification of a recrudescence as a new infection and underestimating the amount of drug resistance in the patient population [30]. The argument for analysing genotypes from Day 1 as well as Day 0 samples for comparison with $D_{f}$ has been made previously and may be particularly valuable in high transmission settings [17,31]. Conversely, due to incomplete immunity resulting from a single treated malaria infection, patients could naturally re-acquire malaria caused by the same parasite genotype if they return to an endemic area with relatively little parasite genetic diversity, this time leading to an over-estimate of drug resistance. Current classification schemes of alleles for use in assigning treatment outcome are limited and often subjective and varying between observers. A recent $\mathrm{MMV} / \mathrm{WHO}$ consensus report on $P$. falciparum genotyping recommends possible use of polyacrylamide gels, capillary electrophoresis, or use of dedicated fragment sizing software (used here), to increase discriminatory power and reduce inconsistencies between clinical trial methodologies [8].

There are several limitations to this analysis. More cases of treatment failure, in particular of new infections with $P$. falciparum would have enabled potential misclassification of outcome to be examined in more detail. Additional emergent minority populations may have been detected if sampling had occurred more frequently than once daily. Band size estimates, while measured by automated reader with as much precision as possible, may also have led to minor inaccuracies. Moreover band 
size variability between the same alleles can potentially occur, leading to false categorization as different alleles. Analysis of samples in this study was limited to once per day so minority parasite clones with only a sporadic and low-level presence in the peripheral circulation compared to the dominant clone(s) could have been missed.

\section{Conclusion}

Limited genotyping of $m s p 1, m s p 2$ and glurp in malaria patients from a localized area of Western Cambodia revealed considerable variation in $m s p 2$ alleles but wellconserved mspl and glurp. Longitudinal genotyping of cases with recurrent malaria during seven days of anti-malarial treatment showed homogeneous genotype dynamics and did not alter the outcome classification compared to conventional genotyping at baseline and $D_{f}$; however it did serve to illustrate some of the inherent complexities when interpreting the parasitological basis of recurrent disease. In contrast to Africa, where malaria transmission rates are higher and artemisinin resistance does not currently appear problematic, lower polyclonality of infection was observed in malaria patients in Cambodia. The exception was the group of recurrent malaria infections in which polyclonality was much higher. The potential role of polyclonal infections in contributing to the spread of artemisinin resistance deserves further investigation.

\section{Competing interests}

The authors declare that they have no competing interests.

\section{Authors' contributions}

DB conceived the study; PG, SS, SW, KP, and ST performed the molecular genetic studies; YS, CL, SS, SD, MF, DS, DB conducted the clinical trial; $\mathrm{PG}, \mathrm{CL}$ and $\mathrm{DB}$ analysed the data; $\mathrm{PG}, \mathrm{CL}, \mathrm{ST}, \mathrm{MS}, \mathrm{CMC}, \mathrm{DW}, \mathrm{MF}$, JM, DS, and DB wrote the manuscript. All authors read and approved the final manuscript.

\section{Acknowledgements}

We thank patients and staff at Tasanh Health Center, Cambodia. We also thank Prof Chris Plowe for helpful comments.

\section{Disclaimer}

The views expressed in this article are those of the authors and do not reflect the official policy of the Department of the Army, Department of Defense, or the U.S. Government.

\section{Author details}

'Department of Immunology and Medicine, Armed Forces Research Institute of Medical Science (AFRIMS), Bangkok, Thailand. ${ }^{2}$ Armed Forces Research Institute of Medical Science (AFRIMS), Phnom Penh, Cambodia. ${ }^{3}$ National Center for Parasitology, Entomology and Malaria Control, Phnom Penh, Cambodia.

Received: 25 July 2013 Accepted: 4 November 2013

Published: 9 November 2013

\section{References}

1. Noedl H, Socheat D, Satimai W: Artemisinin-resistant malaria in Asia. N Engl I Med 2009, 361:540-541.

2. Phyo AP, Nkhoma S, Stepniewska K, Ashley EA, Nair S, McGready R, ler Moo C, Al-Saai S, Dondorp AM, Lwin KM, Singhasivanon P, Day NP, White NJ, Anderson TJ, Nosten F: Emergence of artemisinin-resistant malaria on the western border of Thailand: a longitudinal study. Lancet 2012, 379:1960-1966.

3. WHO: Global Report on Antimalarial Drug Efficacy and Drug Resistance: 2000-2010. Geneva: World Health Organization; 2010.

4. Miotto O, Almagro-Garcia J, Manske M, Macinnis B, Campino S, Rockett KA, Amaratunga C, Lim P, Suon S, Sreng S, Anderson JM, Duong S, Nguon C, Chuor CM, Saunders D, Se Y, Lon C, Fukuda MM, Amenga-Etego L, Hodgson AV, Asoala V, Imwong M, Takala-Harrison S, Nosten F, Su XZ, Ringwald P, Ariey F, Dolecek C, Hien TT, Boni MF, et al: Multiple populations of artemisinin-resistant Plasmodium falciparum in Cambodia. Nat Genet 2013, 45:648-655.

5. Takala-Harrison S, Clark TG, Jacob CG, Cummings MP, Miotto O, Dondorp AM, Fukuda MM, Nosten F, Noedl H, Imwong M, Bethell D, Se Y, Lon C, Tyner SD, Saunders DL, Socheat D, Ariey F, Phyo AP, Starzengruber $P$, Fuehrer HP, Swoboda P, Stepniewska K, Flegg J, Arze C, Cerqueira GC, Silva JC, Ricklefs SM, Porcella SF, Stephens RM, Adams M, et al: Genetic loci associated with delayed clearance of Plasmodium falciparum following artemisinin treatment in Southeast Asia. Proc Natl Acad Sci U S A 2013, 110:240-245.

6. Snounou G, Beck HP: The use of PCR genotyping in the assessment of recrudescence or reinfection after antimalarial drug treatment. Parasitol Today 1998, 14:462-467.

7. Collins WJ, Greenhouse B, Rosenthal PJ, Dorsey G: The use of genotyping in antimalarial clinical trials: a systematic review of published studies from 1995-2005. Malar J 2006, 5:122.

8. WHO: Methods and Techniques for Clinical Trials on Antimalarial Drug Efficacy: Genotyping to Identify Parasite Populations. Geneva, Switzerland: World Health Organization; 2008.

9. Snounou G: Genotyping of plasmodium spp. Nested PCR. Methods Mol Med 2002, 72:103-116.

10. Snounou G, Singh B: Nested PCR analysis of plasmodium parasites. Methods Mol Med 2002, 72:189-203.

11. Viriyakosol S, Siripoon N, Petcharapirat C, Petcharapirat P, Jarra W, Thaithong S, Brown KN, Snounou G: Genotyping of Plasmodium falciparum isolates by the polymerase chain reaction and potential uses in epidemiological studies. Bull World Health Organ 1995, 73:85-95.

12. Snounou G, Xinping Z, Napaporn S, Jarra W, Thaithong S, Brown KN, Viriyakosol S: Biased distribution of msp1 and msp2 allelic variants in Plasmodium falciparum populations in Thailand. Trans R Soc Trop Med Hyg 1999, 93:369-374.

13. Noedl H, Se Y, Sriwichai S, Schaecher K, Teja-Isavadharm P, Smith B, Rutvisuttinunt W, Bethell D, Surasri S, Fukuda MM, Socheat D, Chanthap L: Artemisinin resistance in Cambodia: a clinical trial designed to address an emerging problem in southeast Asia. Clin Infect Dis 2010, 51:e82-e89.

14. Bethell D, Se Y, Lon C, Tyner S, Saunders D, Sriwichai S, Darapiseth S, Teja-Isavadharm P, Khemawoot P, Schaecher K, Ruttvisutinunt W, Lin J, Kuntawungin W, Gosi P, Timmermans A, Smith B, Socheat D, Fukuda MM: Artesunate dose escalation for the treatment of uncomplicated malaria in a region of reported artemisinin resistance: a randomized clinical trial. PLoS One 2011, 6:19283.

15. WHO: Methods for Surveillance of Antimalarial Drug Efficacy. Geneva: World Health Organization; 2009.

16. Färnert A, Snounou G, Rooth I, Björkman A: Daily dynamics of Plasmodium falciparum subpopulations in asymptomatic children in a holoendemic area. Am J Trop Med Hyg 1997, 56:538-547.

17. Nsobya SL, Kiggundu M, Joloba M, Dorsey G, Rosenthal PJ: Complexity of Plasmodium falciparum clinical samples from Uganda during short-term culture. J Infect Dis 2008, 198:1554-1557.

18. Liljander A, Bejon P, Mwacharo J, Kai O, Ogada E, Peshu N, Marsh K, Farnert $A$ : Clearance of asymptomatic $P$. falciparum infections interacts with the number of clones to predict the risk of subsequent malaria in Kenyan children. PLoS One 2011, 6:e16940.

19. Ekala MT, Jouin H, Lekoulou F, Issifou S, Mercereau-Puijalon O, Ntoumi F: Plasmodium falciparum merozoite surface protein 1 (MSP1): genotyping and humoral responses to allele-specific variants. Acta Trop 2002, 81:33-46.

20. Henning L, Schellenberg D, Smith T, Henning D, Alonso P, Tanner M, Mshinda H, Beck HP, Felger I: A prospective study of Plasmodium falciparum multiplicity of infection and morbidity in Tanzanian children. Trans R Soc Trop Med Hyg 2004, 98:687-694.

21. Montgomery J, Milner DA Jr, Tse MT, Njobvu A, Kayira K, Dzamalala CP, Taylor TE, Rogerson SJ, Craig AG, Molyneux ME: Genetic analysis of 
circulating and sequestered populations of Plasmodium falciparum in fatal pediatric malaria. J Infect Dis 2006, 194:115-122.

22. Juliano JJ, Ariery F, Sem R, Tangpukdee N, Krudsood S, Olson C, Looareesuwan S, Rogers WO, Wongsrichanalai C, Meshnick SR: Misclassification of drug failures in Plasmodium falciparum clinical trials in Southeast Asia. J Infect Dis 2009, 200:624-628.

23. Kwiek JJ, Alker AP, Wenink EC, Chaponda M, Kalilani LV, Meshnick SR: Estimating true antimalarial efficacy by heteroduplex tracking assay in patients with complex Plasmodium falciparum infections. Antimicrob Agents Chemother 2007, 51:521-527.

24. Teuscher F, Gatton ML, Chen N, Peters J, Kyle DE, Cheng Q: Artemisinin-induced dormancy in Plasmodium falciparum: duration, recovery rates, and implications in treatment failure. J Infect Dis 2010, 202:1362-1368.

25. Teuscher F, Chen N, Kyle DE, Gatton ML, Cheng Q: Phenotypic changes in artemisinin-resistant Plasmodium falciparum lines in vitro: evidence for decreased sensitivity to dormancy and growth inhibition. Antimicrob Agents Chemother 2012, 56:428-431.

26. Jafari S, Le Bras J, Bouchaud O, Durand R: Plasmodium falciparum clonal population dynamics during malaria treatment. J Infect Dis 2004, 189:195-203.

27. Färnert A, Björkman A: Limited advantage of multiple consecutive samples for genotyping Plasmodium falciparum populations during the first days of treatment. Am J Trop Med Hyg 2005, 73:204-206.

28. Jarra W, Snounou G: Only viable parasites are detected by PCR following clearance of rodent malarial infections by drug treatment or immune responses. Infect Immun 1998, 66:3783-3787.

29. Zimmerman PA, Mehlotra RK, Kasehagen LJ, Kazura JW: Why do we need to know more about mixed Plasmodium species infections in humans? Trends Parasitol 2004, 20:440-447

30. Slater M, Kiggundu M, Dokomajilar C, Kamya MR, Bakyaita N, Talisuna A, Rosenthal PJ, Dorsey G: Distinguishing recrudescences from new infections in antimalarial clinical trials: major impact of interpretation of genotyping results on estimates of drug efficacy. Am J Trop Med Hyg 2005, 73:256-262.

31. Martensson A, Ngasala B, Ursing J, Isabel Veiga M, Wiklund L, Membi C, Montgomery SM, Premji Z, Färnert A, Björkman A: Influence of consecutive-day blood sampling on polymerase chain reaction-adjusted parasitological cure rates in an antimalarial-drug trial conducted in Tanzania. J Infect Dis 2007, 195:597-601.

doi:10.1186/1475-2875-12-403

Cite this article as: Gosi et al:: Evaluation of parasite subpopulations and genetic diversity of the $m s p 1, m s p 2$ and glurp genes during and following artesunate monotherapy treatment of Plasmodium falciparum malaria in Western Cambodia. Malaria Journal 2013 12:403.

\section{Submit your next manuscript to BioMed Central and take full advantage of:}

- Convenient online submission

- Thorough peer review

- No space constraints or color figure charges

- Immediate publication on acceptance

- Inclusion in PubMed, CAS, Scopus and Google Scholar

- Research which is freely available for redistribution 